\title{
ВЫБОР СТРУКТУРЫ КАПИТАЛА КОМПАНИЯМИ \\ НА РАЗВИВАЮЩИХСЯ РЫНКАХ \\ С УЧЕТОМ БИЗНЕС-ЦИКЛОВ ЭКОНОМИКИ
}

\author{
М.С. Кокорева ${ }^{1}$, М.С. Никифоров ${ }^{2}$
}

В данной статье представлены результаты исследования структуры капитала компаний, функциоэнирующих на развивающихся рынках капитала, с учетом бизнес циклов экономики. Проведенное чисследование на данных 581 компаний из стран БРИКС за 2002-2014 гг. позволил установить, что

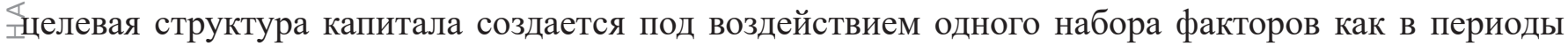
Ғэкономического роста, так и в периоды спада. Скорость приспособления к целевой структуре капиштала при этом зависит от стадии бизнес цикла экономики: в период экономического роста скорость Пприспособления выше. В ходе исследования также выявлено, что направление влияния детермиэнант скорости приспособления меняется в зависимости от состояния экономики (отклонение от делевого показателя структуры капитала приводит к росту скорости приспособления в периоды Пподъема и к падению - в периоды экономического спада).

Ключевые слова: динамическая компромиссная теория, переключающиеся режимы, развивающиеся рынки капитала, структура капитала, скорость приспособления

JEL: G32

\section{Введение}

Выбор оптимального соотношения между собственными и заемными источниками финансироваㄱя является одним из важнейших финансово-управленческих решений компании. Изучению данЧной проблематики посвящено большое количество теоретических и эмпирических исследований خेкак на развитых, так и на развивающихся финансовых рынках. В условиях современных кризисных œялений вопрос о влиянии макроэкономических условий на выбор структуры капитала, получил новое развитие.

Вклад данной работы в исследования структуры капитала на развивающихся финансовых рынках обусловлен в первую очередь недостатком аналогичных исследований, анализирующих процесс формирования структуры капитала компаний в различных макроэкономических условиях. Связано это со спецификой функционирования фирм на развивающихся рынках, которая требует внесения модификаций в обычные модели, а также с недостаточным объемом доступной информации по компаниям до недавнего времени.Второй, и наиболее важный аспект заключается в текущем мировом финансовом кризисе, начавшемся в 2007-2008 гг. Во многих работах работах [Bhamra, Kuehn, Strebulaev, 2010; Hackbarth, 2006] было доказано, что макроэкономическая ситуация в стране способна изменить предпочтения менеджмента компаний по поводу целевой структуры капитала. Но большинство исследований на данную тематику никак не учитывают данный факт. Чаще всего в работах, посвященных детерминантам оптимальной структуры капитала, предполагается, что кризисные данные искажают результаты, ввиду чего данный период исключается из анализа. Однако учитывая периодичность кризисных явлений, периодов экономического спада и роста, очевидна необходимость анализа формирования целевой структуры капитала на разных стадиях экономических бизнес-циклов. Данное исследование исходит из предпосылки, согласно которой при кризисных явлениях в экономике механизмы формирования структуры капитала внутри компаний меняются. Выявление изменений в выборе структуры капитала, связанных с кризисными периодами, является основной задачей работы. Реализованный в данном исследовании метод, подразумевающий использование динамической компромиссной модели структуры капитала с переключением режимов, не только позволяет оценить обозначенные изменения, но и является во многом инновационным для работ, проведенных на данных развивающихся финансовых рынков.

ㄴ

1. Кандидат экономических наук, научный сотрудник НУЛ Корпоративных финансов НИУ ВШЭ.

16. Выпускник магистерской программы «Стратегическое управление фирмы», НУЛ Корпоративных финансов НИУ ВШЭ. 
Исследования динамической компромиссной концепции: детерминанты целевого уровня структуры капитала и скорости приспособления

亡Динамическая компромиссная концепция в настоящее время является ключевой теорией, описываح ющей формирование структуры капитала компаний. Данная концепция подразумевает, что соотно-шение собственного и заемного капиталов фирмы является следствием постоянно осуществляемой Чполитики финансирования данной фирмы, взвешивающей предельные выгоды и предельные издержки, связанные с долговым финансированием, с целью максимизации стоимости фирмы.

Моделирование динамической компромиссной концепции выбора структуры капитала предполаШгает введение в анализ ряда предпосылок. Базовая эконометрическая модель динамической комг промиссной концепции представлена следующей моделью частичного приспособления (уравнение $\ulcorner(1))$ :

$L_{i t}-L_{i-1}=a_{0}+\delta_{i} \cdot\left(L_{i}^{*}-L_{i-1}\right)+\eta_{i}$

где $L-$ показатель долговой нагрузки, $L^{*}-$ целевой уровень долговой нагрузки, $\delta$ - скорость при( ) способления к целевому показателю долговой нагрузки. Основные элементы моделирования включают в себя показатель целевого уровня долговой нагрузки и скорости приспособления. Целевой уровень долговой нагрузки зависит от набора детерми

$L_{i t}=\alpha_{0}=+\sum_{j} \alpha_{j} X_{i j t}$

где Xit - вектор независимых детерминант, оказывающих воздействие на целевой уровень долговой нагрузки.

Для определения целевого показателя долговой нагрузки используется большое число различных детерминант. Но существуют несколько традиционных детерминант, описание которых представлено в таблице 1.

Таблица 1

Традиционные детерминанты финансового рычага.

\begin{tabular}{|c|c|c|}
\hline Детерминанта & Методы определения & Примеры исследований \\
\hline Доходность капитала & EBITDA/Total assets, & Rajan, Zingales, 1995 \\
& ROA & Delcour, 2007 \\
\hline & Pandey, 2001; & Chen, 2004 \\
(Fixed assets) / (Total assets), & Delcoure, 2007 \\
Структура активов & PPE / (Total assets) & Rajan, Zingales, 1995 \\
\hline & & Chen, 2004 \\
& Ln(Sales), & Bhaduri, 2002 \\
& Ln(Assets) & Rajan, Zingales, 1995 \\
Размер капитала компании & & Frank, Goyal, 2008 \\
\hline Налоговый щит & Céspedes et al., 2010 \\
\hline Недолговой налоговый щит & Depreciation / (Total assets) & Chakrabotry, 2010 \\
(NDTS) & Salim et al., 2012 \\
\hline \multirow{2}{*}{ Возможности роста } & Capex / (Total assets) & Mitton, 2008 \\
& Market-to-Book Ratio & Frank, Goyal, 2003 \\
\hline Риск & (Sales growth) / (Total assets) & Wiwattanakantang, 1999 \\
& s. d. (ROA), & Mazur, 2007 \\
\hline
\end{tabular}

В условиях асимметрии информации и несовершенства финансового рынка процесс корректировки структуры капитала может происходить не мгновенно и не полностью. Невозможность мгновенной 
подстройки финансового рычага обусловлена неопределенностью, с которой сталкивается фирма, и транзакционными издержками приспособления (затраты на принятие решения о выпуске ценных бумаг, оплата услуг финансовых посредников и т.д.). Таким образом, параметр подстройки $\delta$ it представляет собой скорость приспособления к оптимальному уровню долговой нагрузки. Если параметр меньше единицы, то компания адаптирует текущий уровень долговой нагрузки к опти৩мальному с задержкой, обусловленной какими-либо факторами. Если больше единицы - компания ¡производит слишком сильную корректировку структуры капитала, в результате чего она снова находится не на своем оптимальном уровне.

Определение детерминант скорости приспособления является отдельным направлением исследований. Скорость приспособления определяется на основе расчётного целевого и фактического (наблюдаемого) уровней долговой нагрузки по формуле 3:

$\delta_{i t}=\frac{L_{i t}-L_{i t-1}}{L_{i t}^{*}-L_{i t-1}}$

Для определения факторов, которые оказывают влияние на скорость приспособления, используется следующее уравнение:

$\delta_{i t}=\beta_{0}+\sum_{k} \beta_{k} Z_{i k t}$

${ }_{\curvearrowright}$ где $Z_{i k t}$-детерминанта $\mathrm{k}$ скорости приспособления для $i$-й компании в момент $t$.

бВ качестве независимых переменных, влияющих на скорость приспособления, можно выделить следующие.

인

○ेОтлонение от целевого уровня $\left(L_{i t}^{*}-L_{i t}\right)$. Предполагается, что если уровень долговой нагрузки неЧзначительно отклоняется от целевой структуры капитала, то у нее нет мотивов к немедленной пере두ойке. Данная детерминанта частично отражает эффект наличия постоянных издержек адаптаळции структуры капитала. Некоторые исследования [Drobetz, Wanzenried, 2006; Banerjee, Heshmati, Wihlbor, 2001] являются примерами работ, в которых было получено подтверждение положительной связи этих показателей, однако в работе Данг с соавторами [Dang et al., 2012] был получен обратный результат - отрицательная зависимость между скоростью приспособления и отклонением от оптимального уровня.

Во-вторых, это размер капитала компании. В соответствии с отдельными работами [Drobetz, Wanzenried, 2004; Mahakud, Mukherjee, 2011; Banerjee, Heshmati, Wihlbor, 2001] данная детерминанта имеет положительную связь со скоростью приспособления. Как правило крупные компании диверсифицированы, а значит, имеют меньшие риски и, соответственно, проще привлекают дополнительное финансирование. Крупным компаниям свойственны низкие постоянные издержки корректировки структуры капитала.

Последний классический фактор - это показатели роста компании. Авторы исследования [Banerjee, Heshmati, Wihlbor, 2001] говорят о том, что компании, демонстрирующие более высокие показатели роста, находятся в лучшем положении для изменения структуры капитала. В первую очередь это связано с естественным ростом собственного капитала компании и большей доступностью заемного финансирования.

Помимо перечисленных выше есть и ряд других детерминант. Например, в исследованиях [Drobetz, Wanzenried, 2006; Öztekin, Flannery, 2012] анализируется влияние институциональных и макроэкономических факторов на скорость приспособления, некоторые из них оказались статистически значимыми.

Среди исследований, изучающих структуру капитала компании в динамике и использующих при этом модели с переключением режимов, можно выделить два основных направления. Первое - paботы, в которых используются модели с пороговыми эффектами (threshold regression models). Их основная идея заключается в том, что можно проследить изменения оценок коэффициентов регрессий при различных уровнях этих же коэффициентов. Например, с помощью подобного метода можно выявить различия в механизме формирования структуры капитала для крупных и небольших компаний (параметр «размер компании» больше или меньше некоторого порогового значе- 
ния, оцениваемого самой моделью). Одним из основных и наиболее известных исследований здесь является работа Дан, Ким, Шин [Dang, Kim, Shin, 2012]. Авторы разработали эконометрическую модель для оценки скорости приспособления к оптимальной структуре капитала фирм, при этом учитывались различия для компаний с высокими и низкими уровнями долговой нагрузки, инвестичцй, волатильности доходов и пр. Другим примером является исследование по тайваньским компа৩ ниям [Lin, Chang, 2009]. Однако оно посвящено не определению детерминант целевой структуры - капитала, а выявлению взаимосвязи между стоимостью компании и оптимальным уровнем долгохвой нагрузки. При этом переключение режимов в модели происходит при пересечении пороговых 夌эффектов для показателя Q Тобина и значения финансового рычага.

${ }^{\ominus}$ Второе направление - модели с переключающимися режимами (regime-switching models), представляющими из себя различные состояния окружающей среды. В отличие от предыдущего типа моделей, здесь переключение режимов происходит не для каждой компании отдельно, а сразу для всех, в соответствии с изменением макроэкономической ситуации. В качестве примеров здесь мож○н привести работы [Hess, Immenkotter, 2011; Bhamra, Kuehn and Strebulaev, 2010; Hackbarth et al., ᄃ 2006]. Данные исследования объединяет наличие в моделях двух режимов, которые соответствуют хдвум состояниям окружающей среды: бум и рецессия. При этом моделируются будущие денежные (?) потоки компаний, оценивается зависимость структуры капитала от показателя прибыли до выпла$\stackrel{\sim}{\neg}$ ты налогов и процентов (ЕВIT). Во всех исследованиях были получены статистически различные ^режимы модели, что подтверждает значимость влияния макроэкономической ситуации на процесс - формирования структуры капитала. В работе [Hess, Immenkotter, 2011] было обнаружено, что изФдержки приспособления к оптимальной структуре капитала выше в период рецессии. Во время †роста экономики компании подстраиваются чаще и при меньших изменениях финансового рычага, при этом демонстрируя более высокую скорость приспособления.

В исследованиях [Hackbarth et al., 2006; Dang, Kim and Shin, 2012; Hess, Immenkotter, 2011] механизм переключения режимов реализован с помощью марковских цепей. Вероятность каждого режима в текущий момент определяется на основе информации о том, в каком режиме находилась модель в предыдущие периоды.

В рамках данной работы будет реализован аналогичный подход, сочетающий в себе традиционную динамическую компромиссную концепцию со скоростью приспособления и бизнес циклы экономики. Ключевое отличие от вышеперечисленных исследований заключается в использовании классических детерминант скорости приспособления к оптимальной структуре капитала, коэффициенты при которых зависят от текущего режима модели. В качестве наиболее наглядного параметра, отражающего стадию бизнес-цикла экономики, в данном исследовании применяется темп роста ВВП. В ходе исследования сформулированы соответствующие гипотезы.

Гипотеза 1: Процесс формирования структуры капитала компаний, функционирующих на рынках стран БРИКС, может быть описан динамической компромиссной концепцией.

Гипотеза 2: Целевой уровень долговой нагрузки зависит от набора характеристик компании (исследуемых детерминант). Предполагаемое влияние детерминант на уровень долговой нагрузки следующее (табл. 2):

Таблица 2

Предполагаемое влияние факторов на уровень долговой нагрузки

\begin{tabular}{|c|c|c|c|}
\hline Детерминанта & Способ расчета & $\begin{array}{c}\text { Предполагаемое влияние на уровень } \\
\text { долговой нагрузки }\end{array}$ & Примеры исследований \\
\hline Доходность капитала & $\begin{array}{c}\text { ROA = Net income / Total } \\
\text { assets }\end{array}$ & $\begin{array}{c}\text { - (доступность привлечения } \\
\text { собственных средств) }\end{array}$ & [Myers, Majluf, 1984] \\
\hline Структура активов & Fixed assets / Total assets & $\begin{array}{c}+ \text { (меньшие издержки финансовой не- } \\
\text { устойчивости, обеспечение долга) }\end{array}$ & $\begin{array}{c}\text { [Galai, Masulis, 1976; Jensen, } \\
\text { Meckling, 1976; Titman, Wessels, } \\
\text { 1988] }\end{array}$ \\
\hline $\begin{array}{c}\text { Размер капитала } \\
\text { компании }\end{array}$ & In(Revenue) & $\begin{array}{c}+ \text { (меньшие издержки финансовой } \\
\text { неустойчивости и более высокие } \\
\text { налоговые выгоды) }\end{array}$ & $\begin{array}{c}\text { [Beck et al., 2008; Titman, } \\
\text { Wessels, 1988] }\end{array}$ \\
\hline
\end{tabular}




\begin{tabular}{|c|c|c|c|}
\hline Налоговый щит & $\begin{array}{c}\text { (Tax Paid) / (Earnings before } \\
\text { tax })\end{array}$ & + (более высокие налоговые выгоды) & $\begin{array}{l}\text { [Modigliani, Miller, 1958; } \\
\text { Adedeji, 2002] }\end{array}$ \\
\hline $\begin{array}{c}\text { Недолговой } \\
\text { налоговый щит } \\
\text { (NDTS) }\end{array}$ & $\mathrm{DA} / \mathrm{TA}$ & $\begin{array}{c}\text { - (заменитель для долговых налоговых } \\
\text { выгод) }\end{array}$ & $\begin{array}{l}\text { [Modigliani, Miller, 1958; } \\
\text { Adedeji, 2002] }\end{array}$ \\
\hline Возможности роста & $\begin{array}{c}\text { Market value of TA / Book } \\
\text { value of TA, a также CAPEX } \\
\text { / Total assets }\end{array}$ & $\begin{array}{c}\text { - (высокие издержки финансовой не- } \\
\text { устойчивости) }\end{array}$ & $\begin{array}{l}\text { [Rajan and Zingales, 1995; } \\
\text { Myers, Majluf, 1984] }\end{array}$ \\
\hline Риск & s. d. (ROA) & $\begin{array}{c}\text { - (высокие издержки финансовой } \\
\text { неустойчивости) }\end{array}$ & [Pandey, 2001; Mazur, 2007] \\
\hline
\end{tabular}

Следующий блок гипотез опирается на выводы предыдущих исследований по структуре капитала, ш которых анализируются изменения макроэкономических условий [Hackbarth, Miao and Morellec, 2004; Dieter, and Immenkotter, 2012].

Гипотеза 3: Издержки приспособления к оптимальной структуре капитала ненулевые, выше в период рецессии, и зависят от макроэкономической ситуации в стране, в частности от темпов роста валового внутреннего продукта (ВВП).

Последняя гипотеза основана на исследования скорости приспособления и проверяется отдельно для каждого режима модели (стадии бизнес-цикла экономики).

Гипотеза 4: Скорость приспособления к оптимальной структуре капитала зависит от набора характеристик компании. Согласно динамической компромиссной концепции, детерминанты должны локазывать на скорость приспособления к оптимальному уровню долговой нагрузки влияние в соот-ветствии с таблицей 3.

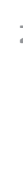

Таблий 3

Предполагаемое влияние факторов на скорость приспособления, в соответствии с динамической компромиссной концепцией

\begin{tabular}{|c|c|c|c|}
\hline Детерминанта & Способ расчета & $\begin{array}{c}\text { Предполагаемое влияние на } \\
\text { скорость приспособления }\end{array}$ & Примеры исследований \\
\hline $\begin{array}{c}\text { Отклонение от целевого } \\
\text { уровня }\end{array}$ & $\begin{array}{c}\text { Разница между } \\
\text { фактическим и целевым } \\
\text { уровнями долговой } \\
\text { нагрузки }\end{array}$ & $\begin{array}{c}\text { + (рост издержек при не } \\
\text { оптимальной СК, наличие } \\
\text { постоянных издержек } \\
\text { корректировки СК) }\end{array}$ & $\begin{array}{l}\text { [Drobetz, Wanzenried, 2006; } \\
\text { Banerjee, Heshmati, Wihlbor, } \\
\text { 2001] }\end{array}$ \\
\hline Размер компании & $\ln$ (Revenue) & $\begin{array}{c}\text { + (ниже относительные издержки } \\
\text { приспособления к оптимальной } \\
\text { структуре капитала) }\end{array}$ & $\begin{array}{l}\text { [Drobetz, Wanzenried, 2004; } \\
\text { Mahakud, Mukherjee, 2011; } \\
\text { Banerjee, Heshmati, Wihlbor, } \\
\text { 2001] }\end{array}$ \\
\hline Возможности роста & $\begin{array}{c}\text { Market value of TA / Book } \\
\text { value of TA, a также CAPEX } \\
\text { / Total assets }\end{array}$ & $\begin{array}{l}\text { + (больше возможностей для из- } \\
\text { менения структуры капитала, за } \\
\text { счет роста) }\end{array}$ & $\begin{array}{l}\text { [Banerjee, Heshmati, Wihlbor, } \\
\text { 2001] }\end{array}$ \\
\hline Структура активов & Fixed assets / Total assets & $\begin{array}{c}\text { + (проще и выгоднее привлекать } \\
\text { заемные средства в связи с } \\
\text { наличие обеспечения) }\end{array}$ & - \\
\hline Структура долга & $\begin{array}{l}\text { (Short-term borrowings) / } \\
\text { (Total debt) }\end{array}$ & $\begin{array}{c}\text { - (компании с большей долей долго- } \\
\text { срочных заимствований обладают } \\
\text { меньшей гибкостью в корректиров- } \\
\text { ке СК) }\end{array}$ & - \\
\hline
\end{tabular}

\section{Методология}

Исследование динамической компромиссной концепция со скоростью приспособления с использованием переключающихся режимов проводится в три этапа. На первом этапе проводится оценка коэффициентов при детерминантах фактического уровня долговой нагрузки (оцениваются коэффициенты $\alpha \_j$ из уравнения (2)) для определения целевого уровня долговой нагрузки. На втором этапе, используя фактический и полученный ранее целевой уровни долговой нагрузки, в соответствии с формулой (3) рассчитывается скорость приспособления - $\delta_{i t}$. Последний этап - оценка влияния на скорость приспособления соответствующих детерминант (коэффициенты $\beta_{k}$ из уравнения (4)). 
Особенностью данного исследования в первую очередь является использование регрессионных моделей для панельных данных с реализованным механизмом переключения режимов.

Эконометрическая методология переключения режимов в регрессионном уравнении, используемая в данном исследовании, основана на работах [Goldfeld and Quandt, 1973] и [Asea, Blomberg, 1998].

글 рамках модели предполагается, что текущее состояние экономики (окружающей среды), от кото-рого зависят мотивы принятия решений руководством компаний, можно описать с помощью ряда чдетерминант. Тогда механизм переключения в модели в наиболее простом случае с двумя режимами будет контролироваться линейным логистическим уравнением с регрессорами $\mathrm{z}_{\mathrm{t} 1}, \ldots, \mathrm{z}_{\mathrm{tL}}$ :

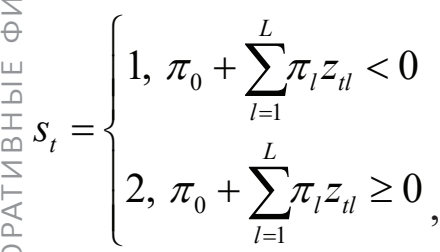

где $s_{t}$ - переменная, задающая текущее состояние окружающей среды,

$\pi_{1}-$ неизвестные коэффициенты.

Пусть экономика страны может находиться в двух состояниях:

○् При $s_{t=1}$ - бум. Характеризуется ростом ВВП.

бٓПри $s_{t=2}$ - рецессия/стагнация. Характеризуется падением или сохранением текущего уровня ВВП.

m Таким образом, вероятности режимов заданы с помощью вспомогательной регрессии от темпов 으ироста ВВП России с учетом фиксированных эффектов, свойственным компаниям. Предполачгается, что ошибки модели распределены нормально, и их дисперсия может зависеть от режима. ¿Тогда регрессионное уравнение можно записать как:

$y_{i t}=\beta_{1, i}(j)+\sum_{k=2}^{K} \beta_{k}(j) x_{k i t}+\varepsilon_{i t}(j) \quad$ for $s_{t}=j$,

$\varepsilon_{i t}(j) \sim N\left(0, \sigma^{2}(j)\right)$

$\operatorname{prob}\left(s_{t}=j\right)=p_{i j}$

где $y_{i t}$-зависимая переменная для $i$-й компании в момент времени $\mathrm{t}$

$x_{i t}-$ независимая переменная $\mathrm{k}$ для $i$-й компании в момент времени $\mathrm{t}$.

Функция плотности распределения для переменной имеет вид:

$p_{t}\left(y_{i t}, X_{i t}\right)=\frac{1}{\sqrt{2 \pi} \sigma\left(s_{t}\right)} \exp \left\{-\frac{1}{2 \sigma^{2}\left(s_{t}\right)}\left[y_{i t}-X_{i t}{ }^{\prime} \beta\left(s_{t}\right)\right]^{\prime}\left[y_{i t}-X_{i t}{ }^{\prime} \beta\left(s_{t}\right)\right]\right\}$.

Функция вероятности нахождения мира в определенном режиме имеет мультиномиальную логистическую спецификацию с константой и одним регрессором - прирост ВВП за период. Соответственно вероятность наступления режима в конкретный период является функцией от линейного уравнения с рядом собственных детерминант:

$P\left(s_{t}=1\right)=f(q)$

$f(q)=\frac{1}{1+e^{-q}}$

$q=\pi_{0}+\sum_{l=1}^{L} \pi_{l} z_{t l}$

Как видно в равенствах (8-10), рассчитав коэффициенты при детерминантах режима, можно найти вероятность нахождения в определенном состоянии $f(q)$ в момент $t$.

Логарифмическая функция правдоподобия, получаемая взвешенным по вероятностям суммированием функций из уравнения (5), имеет вид: 
$l(\beta, \pi, \sigma)=\sum_{t=1}^{T} \sum_{i=1}^{N} \log \left\{\sum_{m=1}^{S} p_{t}\left(y_{i t}, X_{i t}\right) P\left(s_{t}=m \mid Z, \pi\right)\right\}$,

где $\mathrm{S}$ - количество режимов в модели $(\mathrm{S}=2)$;

$\stackrel{\sim}{\sim} P\left(s_{t}=m \mid Z, \pi\right)$ - вероятность режима т при определенных векторах $\mathrm{Z}$ и $\pi$.

^Для получения оценок коэффициентов модели необходимо максимизировать функцию (11) по неизвестным параметрам $\pi, \beta$ и $\sigma$.

Данная методология, однако, не позволяет тестировать сразу все потенциально значимые факторы в одном регрессионном уравнении, в связи с чем одной из ключевых задач исследования является швыбор наиболее подходящей спецификации, включающей в себя наибольшее количество значимых 韋детерминант.

\section{Переменные и выборка}

В качестве зависимой переменной финансового рычага в работе использованы два варианта:

- Рыночный уровень долговой нагрузки - (Total debt)/(Total debt + Market value of total equity).

- Балансовый уровень долговой нагрузки - (Total debt)/(Total assets).

Н Набор анализируемых детерминант оптимальной структуры капитала помимо обозначенных в таблице 2 включает в себя дамми-переменные для секторов экономики - 8 секторов в соответствии mc Глобальным стандартом классификации отраслей («GICS»). Сектора идентифицируются по перғвым двум цифрам номера GICS. Набор анализируемых детерминант скорости приспособления к 少целевой структуре капитала представлен в таблице 3.

В работе используются ежеквартальные данные по торгующимся компаниям стран БРИКС с первого квартала 2002 года по четвертый квартал 2014 года. В качестве источников финансовых показателей выступает база данных Блумберг (Bloomberg) и официальные сайты компаний, публикующих свою отчетность. Источниками макроэкономических данных являются также база данных Блумберг и сайт Всемирного банка (The World Bank).

Из выборки были исключены компании финансовых секторов экономики. В связи со специфичностью некоторых показателей и большим количеством пропусков были также исключены компании с объемом доступных данных для менее чем 25 наблюдений (большая их часть является фирмами из Китая). Также почти для всех переменных были применены процедуры «замены» («winsorising») и «отсечения» («trimming») с 2-5\% квантилями для исключения выбросов. Для выполнения расчетов также были исключены компании с нулевым или очень близким к нулю уровнем финансового рычага.

В результате описанных выше действий первоначальная выборка, состоящая из 3000 компаний, сократилась до 581. Модель строится по несбалансированной панели, состоящей из 581 компаний (кросс-секций) и 52 периодов (всего 30212 наблюдений).

На рисунке 1 представлено распределение компаний выборки по странам.

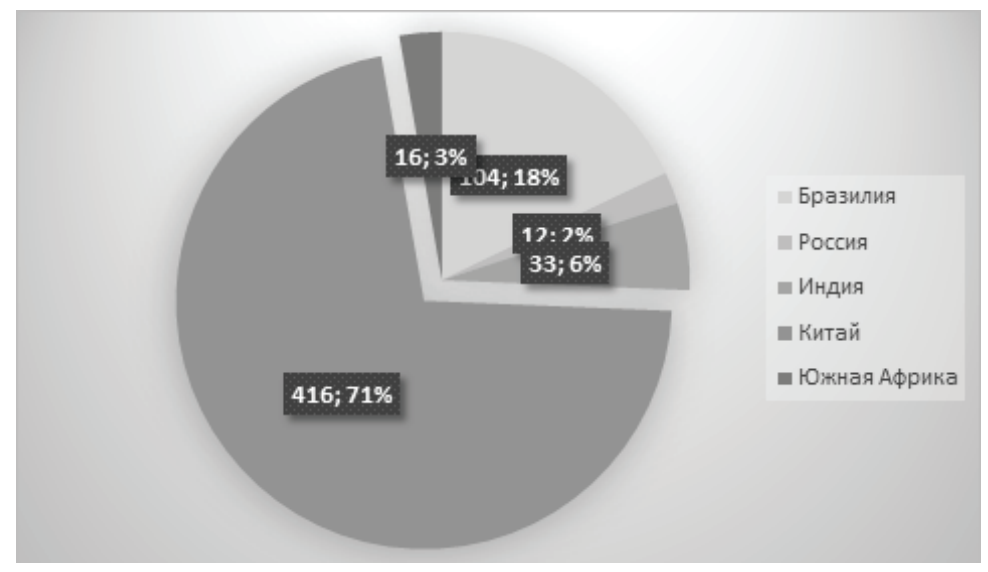

Рисунок 1. Распределение компаний выборки по странам 
Хорошо видно, что большую часть используемых данных составляют фирмы из Китая. Это приводит к сильному смещению выборки в сторону компаний этой страны, и является частым явлением в исследованиях, использующих выборки по странам БРИКС.

\section{Результаты эмпирического тестирования модели формирования структуры капитала с переключением режимов в странах БРИКС}

Два варианта расчёта уровня долговой нагрузки (с использованием балансовой или рыночной стоимости собственного капитала) были рассмотрены поочередно, при этом в качестве основного варианта анализируется показатель, основанный на балансовых величинах собственного капитала. Все расчеты проводились с использованием статистических программных пакетов EViews и R.

\section{Эмпирическое тестирование балансового уровня долговой нагрузки}

На первом этапе оценивался целевой уровень структуры капитала. В итоговую спецификацию модели вошли только 6 статистически значимых детерминант, которые представлены в таблице 4. Также, приведенное тестирование показало отсутствие в данном случае статистически значимых режимов модели. Поэтому оптимальный уровень долговой нагрузки рассчитывается по всем доступным данным и при отсутствии переключения режимов.

Таблий 4

Оценки коэффициентов при детерминантах оптимального уровня долговой нагрузки

\begin{tabular}{|c|c|c|}
\hline Переменные & Коэффициенты & t-статистики \\
\hline Размер компании & $0.817 * * *$ & 8,591 \\
\hline Уровень инвестиций & $-61,099 * * *$ & $-10,623$ \\
\hline ROA & $-1,177 * * *$ & $-40,776$ \\
\hline Структура активов & $2,259 * * *$ & 3,554 \\
\hline Возможности роста & $-1,537 * * *$ & $-17,012$ \\
\hline Эффективность (ОРМ) & $0,203 * * *$ & 15,335 \\
\hline
\end{tabular}

Помимо указанных в таблице 4 переменных, в модель также были включены отраслевые даммипеременные для восьми секторов экономики.

Размер капитала компании оказывает положительное влияние на оптимальный уровень долговой нагрузки. Это соответствует динамической компромиссной концепции, и обуславливается более низкими издержками финансовой неустойчивости у крупных компаний. Уровень инвестиций и возможности роста (Q Тобина) отвечают за перспективы роста собственного капитала компании, и негативно влияют на структуру капитала. Это также соответствует результатам предыдущих исследований [Nivorozhkin, 2002; Chen, 2004]. Отрицательный знак коэффициента при детерминанте доходности капитала компании (ROA) может быть объяснен с точки зрения теории порядка финансирования. Объясняется это тем, что компании, имеющие высокую нераспределенную прибыль вследствие высокой рентабельности, выберут данный внутренний источник средств для финансирования проектов. Структура активов, согласно компромиссной теории, выражает способность компании предоставить залог при предоставлении займа, а соответственно положительно влияет на уровень долговой нагрузки.

Полученные коэффициенты при детерминантах оптимальной структуры капитала соответствуют предполагаемым в гипотезе 2, не считая оказавшихся незначимыми налогового щита и NDTS. Поэтому в целом эту гипотезу нельзя отвергнуть, а результаты совпадают с полученными многими другими авторами в предыдущих исследованиях по статической компромиссной концепции.

Используя полученные коэффициенты при регрессорах уровня долговой нагрузки, в соответствии с уравнением 2 рассчитывается целевая структура капитала. На основе этого показателя далее рассчитывается скорость приспособления к оптимальной структуре капитала и определяются ее де- 
терминанты на разных стадиях бизнес-циклов экономики.

Режимы идентифицируются знаком при коэффициенте у темпов прироста национального ВВП, которые в свою очередь в каждом периоде различаются для компаний из разных стран. Это позволяяет построить единую модель, не создавая страновых подвыборок, а также получить различные ○вероятности режимов для компаний из разных стран. При положительной зависимости, с увеличением этого регрессора растет вероятность нахождения экономики в состоянии подъема (). При 今отрицательной - растет вероятность рецессии или стагнации ().

Таблица 5

Оценки коэффициентов при факторах скорости приспособления

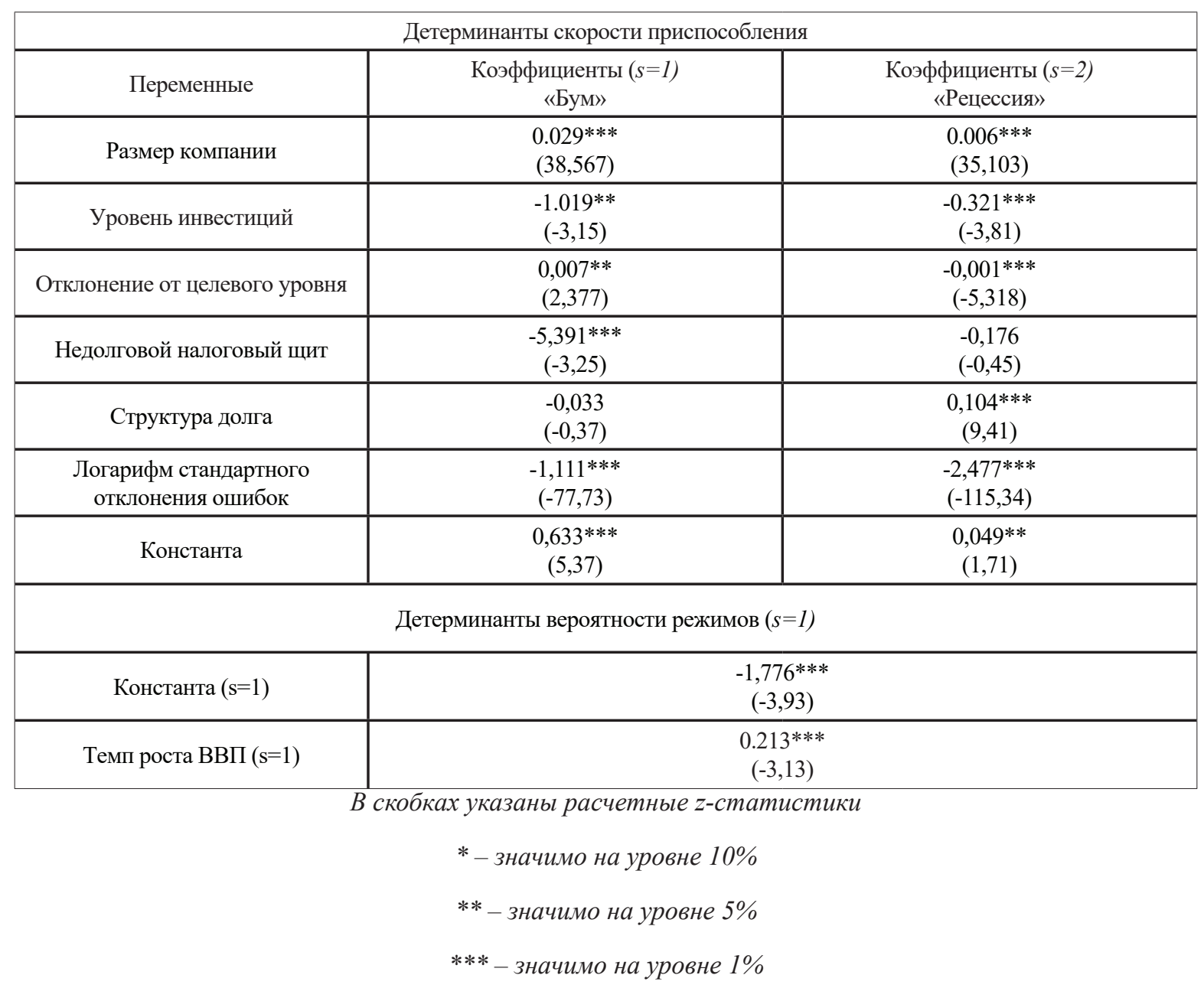

Полученные коэффициенты при детерминантах в итоговой спецификации представлены в таблице 5. Верхняя секция таблицы показывает коэффициенты при детерминантах скорости приспособления, нижняя - при детерминантах параметра , указывающего на режим модели. В качестве базового (), используется режим «бум/рост». Вероятность другого режима («стагнация/рецессии») соответственно высчитывается как:

$P\left(s_{t}=2\right)=1-P\left(s_{t}=1\right)$

При благоприятном состоянии экономики (режим 1), наблюдается статистически значимая положительная связь скорости приспособления с размером компании, отклонением от оптимального левериджа и структуры капитала (доли краткосрочных обязательств в совокупном заемном капитале), что согласуется с выдвинутой ранее гипотезой 2 о предполагаемом влиянии используемых детерминант на оптимальный уровень долговой нагрузки. Отрицательная зависимость с недолговым налоговым щитом и уровнем инвестиций в обоих режимах. Также отклонение от целевого уровня во втором режиме становится отрицательным. Кроме того, в данном случае становятся незначимыми недолговой налоговый щит и структура долга.

Второй режим характеризуется меньшим стандартным отклонением ошибок. Соответственно, ис- 
пользуемые детерминанты лучше описывают скорость приспособления: при ухудшении ситуации в экономике роль случайного фактора уменьшается. В совокупности со значительно более низкой константой и коэффициентами при детерминантах во втором режиме, это свидетельствует о низкой скорости приспособления к оптимальной структуре капитала в данном режиме. Для подтверждеᄂ $\rightarrow$ ния этой гипотезы можно построить простую модель, в которой при переходе при смене режимов ৩меняется только константа и стандартное отклонение ошибок. Тогда скорость приспособления при -благоприятной экономической ситуации в среднем равна 0,57 , против 0,11 и меньшем отклонении 于 ошибок при рецессии. Это не позволяет отвергнуть выдвинутую ранее гипотезу 3 , о более высокой скорости приспособления в первом режиме. Также это соответствует результатам, полученным в $\checkmark$ исследовании [Hackbarth, Miao and Morellec, 2004].

Объяснить данное явление можно высокими издержками изменения структуры капитала в кризисные периоды. Компаниям трудно привлекать дополнительный заемный капитал, при этом возникают проблемы и с погашением уже имеющегося заемного капитала в связи с сократившимися объемами доступных денежных средств. И хотя издержки нахождения не в оптимальном уровне долговой нагрузки в кризис выше, компании предпочитают не вносить в него существенные корхректировки.

(⿹) Приведенные выше результаты получены при оценивании модели на 6668 наблюдениях, что существенно ниже общего числа наблюдений итоговой выборки. Связано это с ее сокращением из-за добавления детерминант, таких как NDTS и уровень инвестиций, объем доступных наблюдений, по бкоторым, существенно ниже. Поэтому результаты были проверены при тестировании сокращенного числа детерминант на большем числе наблюдений, равном 16 тыс.

ㄱри рассмотрении значимых детерминант можно сделать следующие выводы:

Размер капитала компании. Крупные компании обычно диверсифицированы, а значит, в меньшей ᄃстепени подвержены риску банкротства. С одной стороны, издержки финансовой неустойчивости, ${ }^{\infty}$ а соответственно и нахождения не в оптимальном уровне финансовой нагрузки для подобных фирм ниже. С другой - на фоне меньших компаний им проще и дешевле привлекать финансирование при необходимости, особенно в кризисные периоды. В нашем исследовании получено, что крупным компаниям свойственна более высокая скорость приспособления, при этом при благоприятной ситуации в экономике влияние этого фактора на скорость приспособления выше. В условиях кризиса крупные компании, несмотря на более высокую скорость приспособления, предпочитают реже корректировать свой финансовый рычаг.

Возможности роста (уровень инвестиций). Компании, проводящие активную инвестиционную политику, нуждаются в большем финансировании. И, как правило, более быстрым и простым является привлечение заемного капитала. Соответственно, текущая структура капитала данных компаний сформирована не с учетом издержек финансовой неустойчивости и налогового щита, а по необходимости минимальных затрат ресурсов получить необходимое финансирование. Поэтому и скорость приспособления имеет низкие значения.

Отклонение от целевого уровня. В периоды роста национальных экономик данная детерминанта оказывает статистически значимое положительное влияние на скорость приспособления. Это говорит о том, что чем больше отклонение уровня долговой нагрузки компании от оптимального, тем больше у нее стимулов внести корректировки, а соответственно выше скорость приспособления. Косвенно это может свидетельствовать о наличии «горизонта бездействия», элемента другой модели динамической теории формирования структуры капитала. То есть компании не корректируют существенно свой уровень долговой нагрузки, пока отклонение достаточно мало. А при пересечении определенных границ «горизонта бездействия», где издержки нахождения не в оптимальном уровне начинают превышать затраты на его изменение, они производят подстройку сразу к нужному им целевому уровню, и у них резко повышается скорость приспособления.

В кризисный период - напротив, влияние данного фактора на скорость приспособления - отрицательное и существенно ниже, чем в другом режиме. Преимущественно, большое отклонение от оптимального уровня говорит о том, что или у компании проблемы с большой долговой нагрузкой, или она старается не привлекать существенные кредиты. В первом случае, вероятно, у неё нет воз- 
можности оперативно скорректировать свой леверидж с учетом кризиса. Во втором - компания не собирается менять кардинально свою политику финансирования, а соответственно и скорость приспособления у нее низкая (далека от 1).

${ }_{\bigcap}$ Недолговой налоговый щит. Недолговой налоговый щит в обоих режимах оказывает отрицательное ¡ влияние на скорость приспособления, причем в режиме бума это влияние значительно сильнее и только в нем оно статистически значимо. Данный вид уменьшения налогооблагаемой базы за счет

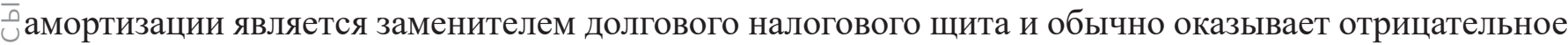
влияние на уровень долговой нагрузки. Поскольку NDTS уменьшает значимость обычного долгового налогового щита для компании, то, возможно, он понижает и значимость оптимальной струк-

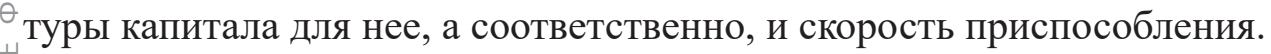

Структура долга. Отношение краткосрочного долга к совокупному заемному капиталу оказывает статистически значимое положительное влияние на скорость приспособления, но только в режиме спада/рецессии. Небольшая доля краткосрочных заимствований у компании указывает на то, что у ᄃнее или в принципе было мало долгосрочных кредитов, или что скоро выплаты по ним прекратятся. ○Таким образом, подобным компаниям проще будет уменьшить свой уровень финансового рычага (скорое погашении действующих кредитов), или, при необходимости, привлечь новые. В режиме бум/рост коэффициент статистически не значим.

○े Получившиеся коэффициенты для первого режима почти полностью соответствуют предполагае-мым в гипотезе 4. Исключением является уровень инвестиций, который выступает в качестве прок๓ си для возможностей роста компании. Во втором режиме ожидаемое влияние оказывает только $\checkmark$ размер фирмы. Связано это в первую очередь с тем, что гипотеза формулировалась на основе пре之дыдущих исследований по динамической компромиссной концепции со скоростью приспособле$\checkmark$ ния, в которых не включались кризисные периоды. В полученной нами модели, это соответствует 元ервому режиму (бум/рост экономики). В другом режиме механизмы адаптации к оптимальному $\curvearrowleft$ уровню структуры капитала меняются, а соответственно и влияние анализируемых факторов на скорость приспособления становится другим.

Гипотеза 3 не может быть отвергнута ввиду наличия статистически различных режимов модели, вероятности которых формируются показателем темпов роста национальных ВВП стран. В совокупности, можно заключить, что процесс формирования структуры капитала компаний, функционирующих на рынках стран БРИКС, может быть описан динамической компромиссной концепцией со скоростью приспособления, механизм формирования которой зависит от текущей стадии бизнес-цикла экономики.

\section{Эмпирическое тестирование рыночного уровня долговой нагрузки}

Исследование проведено согласно той же методологии и последовательности действий, но с использованием другого показателя структуры капитала, рассчитанного на основе рыночной стоимости собственного капитала компаний. В таблице 6 представлены оценки детерминант целевого уровня долговой нагрузки.

Таблица 6

Оценки коэффициентов при детерминантах целевого уровня долговой нагрузки на основе рыночных показателей

\begin{tabular}{|c|c|c|}
\hline Переменные & Коэффициенты & $t$-статистики \\
\hline Размер компании & $0.817 * * *$ & -591 \\
\hline Уровень инвестиций & $-0,292 * * *$ & $-5,74$ \\
\hline RОА & $-0,012 * * *$ & $-46,46$ \\
\hline Структура активов & 0,009 & 1,62 \\
\hline Возможности роста & $-0,049 * * * 2,26$ \\
\hline Эффективность (ОРМ) & $*$ - значимо на уровне 10\% & 15,19 \\
\hline & $* *-$ значимо на уровне 5\% & \\
\hline
\end{tabular}


Содержательно результаты данной регрессии не отличаются от представленных ранее и гипотеза 2 о предполагаемом влиянии детерминант на структуру капитала, также не может быть отвергнута. Исключением стал показатель структуры активов компаний, который при сохранении положительного влияния стал статистически незначимым. Все дамми-переменные, отражающие специфику ᄂ

Вновь отсутствуют статистически значимые различные режимы модели, поэтому оптимальный 总уровень долговой нагрузки рассчитывается по всем доступным данным и при отсутствии переключения режимов.

Следующий этап - оценка влияния детерминант скорости приспособления при различных режишмах. В противоположность коэффициентам, полученным для балансовой структуры капитала, большинство полученных здесь являются статистически незначимыми. При этом различные режимы модели выделяются. Хотя их переключение нельзя однозначно интерпретировать изменением стадии бизнес-цикла, в связи с низкими коэффициентами при факторах вероятности режима и Оильным перекосом в сторону первого режима (бум/рост экономики).

Результаты тестирования спецификации, получившейся наиболее оптимальной в прошлом вари() анте, представлены в таблице 7. Значимое положительное влияние на скорость приспособления показывает только размер компании во втором режиме (спад/рецессия).

Таблица 7

Оценки коэффициентов при факторах скорости приспособления на основе рыночных показателей.

\begin{tabular}{|c|c|c|}
\hline \multicolumn{3}{|c|}{ Детерминанты скорости приспособления } \\
\hline Переменные & $\begin{array}{c}\text { Коэффициенты }(s=1) \\
\text { Бум }\end{array}$ & $\begin{array}{c}\text { Коэффициенты }(s=2) \\
\text { Рецессия }\end{array}$ \\
\hline Размер компании & $\begin{array}{l}0,006 \\
(0,27)\end{array}$ & $\begin{array}{c}0,011 * * * \\
(8,07)\end{array}$ \\
\hline Уровень инвестиций & $\begin{array}{l}-0,232 \\
(-1,38)\end{array}$ & $\begin{array}{l}-0,621 \\
(-0,79)\end{array}$ \\
\hline Недолговой налоговый щит & $\begin{array}{l}0,003 \\
(0,46) \\
\end{array}$ & $\begin{array}{l}1,659 \\
(0,81) \\
\end{array}$ \\
\hline Структура долга & $\begin{array}{l}-0,137 \\
(-1,44)\end{array}$ & $\begin{array}{l}-0,012 \\
(0,59)\end{array}$ \\
\hline $\begin{array}{c}\text { Логарифм стандартного } \\
\text { отклонения ошибок }\end{array}$ & $\begin{array}{c}-2,531 * * * \\
(-17,51)\end{array}$ & $\begin{array}{c}-0,245^{* * *} \\
(-12,67)\end{array}$ \\
\hline Константа & $\begin{array}{c}0,018 * * * \\
(4,48)\end{array}$ & $\begin{array}{l}0,211 * * \\
(13,45)\end{array}$ \\
\hline \multicolumn{3}{|c|}{ Детерминанты вероятности режимов $(s=1)$} \\
\hline Константа $(\mathrm{s}=1)$ & \multicolumn{2}{|c|}{$-1,039 * * *(-6,29)$} \\
\hline Темп роста ВВП (s=1) & \multicolumn{2}{|c|}{$0,026^{* * *}(1,99)$} \\
\hline
\end{tabular}

В скобках указаны расчетные z-статистики

* - значимо на уровне $10 \%$

** - значимо на уровне $5 \%$

***-значимо на уровне $1 \%$

Поставленные в данном исследовании гипотезы в данном случае могут быть отвергнуты. Тестирование средних значений скорости приспособления в каждом режиме показало, что компании быстрее подстраиваются во втором режиме.

Неустойчивые результаты, основанные на данных рыночного финансового рычага, довольно ожидаемы. Как было получено в работе Кокоревой [Кокорева, 2012], компании, функционирующие на развивающихся рынках капитала, определяют в качестве целевых уровней долговой нагрузки показатели, рассчитанные на основе величины совокупного долга и балансовой стоимости собственного капитала. Связано это, в первую очередь, с проблематичностью контроля менеджментом фирм рыночных показателей в условиях нестабильных и слаборазвитых финансовых рынков. Поскольку 
целью данной работы является выявление именно детерминант скорости приспособления к оптимальному уровню долговой нагрузки, и выборка состоит из стран с развивающимися финансовыми рынками, использование балансовых показателей при расчете структуры капитала является предпочтительным.

\section{Заключение}

В данном исследовании было проведено тестирование динамической компромиссной концепции при использовании механизма переключения режимов на данных компаний с развивающихся финансовых рынков. По результатам исследования были сделаны следующие выводы.

Во-первых, было подтверждено влияние на оптимальный уровень структуры капитала компаний на развивающихся рынках традиционных финансовых детерминант: размера капитала компаний, возможностей роста, доходности капитала и структуры активов. При этом наблюдаемые взаимосвязи преимущественно подтверждают компромиссную концепцию формирования структуры капитала.

Во-вторых, были выявлены принципиальные различия в подстройке к оптимальной структуре капиитала компаний стран БРИКС в зависимости от текущей стадии бизнес-цикла экономики. Были ( ) проанализированы два режима модели: бум/рост и стагнация/рецессия. Ключевым фактором, Слияющим на переключение режима, является темп роста национального ВВП. Первый режим ح характеризуется высокими перспективами роста компаний и низкими издержками привлечения خдополнительного финансирования. Второй, в свою очередь, - высокими затратами привлечения эдополнительного финансирования, падением доходов компаний и существенной неопределенно>cтью. Глобальный финансовый кризис, начавшийся в 2007-2008 гг, является наглядным примером второго режима модели. Затраты на корректировку структуры капитала значимо повышаются на фоне издержек нахождения не в оптимальной структуре капитала, что в итоге приводит к снижеСию скорости приспособления.

В-третьих, было выявлено, что у скорости приспособления есть ряд статистически значимых детерминант: размер капитала компаний, отклонение фактической структуры капитала от оптимальной, возможности роста, доходность капитала, недолговой налоговый щит, структура активов и структура заимствований. Причем их влияние меняется в различных стадиях бизнес-цикла экономики. Нам удалось показать, что отклонение фактической структуры капитала от оптимальной в первом режиме оказывает положительное влияние на скорость приспособления, что соответствует результатам многих других исследований, построенных по данным с исключенными периодами финансовой нестабильности (финансовых кризисов), а в неблагоприятном - отрицательное.

Использование моделей с переключающимися режимами позволяют существенно расширить наши представления о формировании структуры капитала на развивающихся финансовых рынках. В качестве дальнейших направлений развития данного исследования можно рассматривать включение других макроэкономических факторов, которые могут определять текущий режим модели для более полного описания макроэкономической ситуации.

\section{Список использованных источников}

1. Asea P. K., Blomberg B. Lending cycles //Journal of Econometrics. - 1998. - T. 83. - №. 1. - C. 89-128.

2. Banerjee S., Heshmati A., Wihlborg C., (2000). The Dynamics of Capital Structure, Economics and Finance No. p. 333

3. Bhaduri S., (2002). N. Determinants of Capital Structure Choice: a Study of the Indian Sector. Applied Financial Economics, 2002, 12, p. 655-665

4. Bhamra S., Kuehn L., Strebulaev I., (2010). The Aggregate Dynamics of Capital Structure and Macroeconomic Risk, Review of Financial Studies 23, 12, p. 4187-4241

5. Céspedes J., Gonzaléz M., Molina C.A. Ownership and capital structure in Latin America. Journal of Business Research, 63, 2010, pp.248-254 
6. Chakraborty I. Capital Structure in an emerging stock market: The case of India. Research in International Business and Finance, 24 (2010), 295-314

7. Chen J.J., (2004). Determinants of Capital Structure of Chinese-listed Companies. Economic Change and Restructuring, Vol. 38 (1), 2004, p. 1-35.

8. Dang, V.A, Kim, M., Shin, Y., (2012). Asymmetric Capital Structure Adjustments: New Evidence from Dynamic Panel Threshold Models. Journal of Empirical Finance, 19(4), p. 465-482.

9. Delcoure N., (2007). The determinants of capital structure in transition economies. International Review of Economics and Finance, 16 (2007), p. 400-415

10. Drobetz, Wolfgang, Wanzenried G., (2006). What determines the speed of adjustment to the target capital structure? Applied Financial Economics 16:13, p. 941-958.

11. Frank M., Goyal V., (2003). Testing the Pecking Order Theory of Capital Structure // Journal of Financial Economics. Vol. 67. N 2., p. 217-248.

12. Frank M.Z., Goyal V.K., (2008). Trade-Off and Pecking Order Theories of Debt. Handbook of Empirical Corporate Finance, Volume 2 Edited by B. Espen Eckbo, 2008, p. 135-202

13. Goldfeld S. M., Quandt R. E. A Markov model for switching regressions //Journal of econometrics. - 1973. - T. 1. - №. 1. - C. 3-15.

14. Hackbarth, D., Miao J., Morellec E., (2006). Capital structure, credit risk, and macroeconomic conditions, Journal of Financial Economics, 82, p. 519 -550.

15. Hess D., Immenkötter P., (2011). Optimal leverage, its benefits, and the business cycle. CFR Working Papers 11-12, University of Cologne

16. Mahakud J., Mukherjee S., (2011). Determinants of Adjustment Speed to Target Capital Structure: Evidence from Indian Manufacturing Firms, 2011 International Conference on Economics and Finance Research IPEDR vol.4

17. Mazur K., (2007). The determinants of capital structure choice: Evidence from Polish Companies, International Atlantic Economic Society, 13, p. 495-514

18. Mitton, T., (2008). Why Have Debt Ratios Increased for Firms in Emerging Markets? European Financial Management, Vol. 14, Issue 1, p. 127-151.

19. Nivorozhkin, E., (2002). Capital Structures in Emerging Stock Market: the Case of Hungary. The Developing Economies Volume 40, Number 2, p. 1-27

20. Öztekin Ö., Flannery M., (2012). Institutional determinants of capital structure adjustment speeds, Journal of Financial Economics 103 (1), p. 88-112

21. Pandey I., (2001). Capital Structure and the Firm Characteristics: Evidence from an Emerging Market, IIMA Working Paper No. 2001-10-04, p. 1-19.

22. Rajan G., Zingales L., (1995). What Do We Know About Capital Structure? Some Evidence from International Data, NBER Working Papers No. 4875, p. 1421-1460.

23. Mahfuzah Salim, Raj Yadav, Capital Structure and Firm Performance: Evidence from Malaysian Listed Companies, Procedia - Social and Behavioral Sciences, Volume 65, 3 December 2012, Pages 156-166, ISSN 1877-0428, http://dx.doi.org/10.1016/j.sbspro.2012.11.105.

24. Wiwattanakantang Y., (1999). An empirical study on the determinants of the capital structure of Thai firms. Pacific-Basin Finance Journal 7 (3-4), p. 371-403.

25. Кокорева М., (2012). Выбор структуры капитала компаниями стран БРИК и Восточной Европы: эмпирический анализ. Корпоративные финансы. 2012. № 2., С. 58-70. 
CAPITAL STRUCTURE CHOICE IN EMERGING MARKETS:

DO BUSINESS CYCLES MATTER?

\author{
Maria Kokoreva, \\ Research Fellow, Corporate Finance Center, \\ National Research University Higher School of Economics,
}

Mikhail Nikiforov,

"Strategic Corporate Finance" master programme, NRU HSE

\begin{abstract}
This article presents the results of a study of corporate capital structure in emerging capital markets, taking into account the business cycles of the economy. Our study was conducted on the data of 581 companies from BRICS countries for the years 2002-2014. We revealed that the target capital structure is dependent on set of factors which is the same for both periods of economic growth and recession. The speed of adjustment to the target capital structure is dependent upon the stage of the business cycle of the economy and is higher for in economic growth periods. The study also found out that the direction of the impact of the determinants of the speed of adjustment varies depending on the state of the economy (the deviation from the target capital structure leads to an increase in the speed of adjustment in periods of growth and a fall - in times of recession).
\end{abstract}

Key words: dynamic capital structure, switching regimes, emerging capital markets, speed of adjustment

JEL: G32

\title{
References
}

1. Asea P. K., Blomberg B. Lending cycles //Journal of Econometrics. - 1998. - T. 83. - №. 1. - C. 89-128.

2. Banerjee S., Heshmati A., Wihlborg C., (2000). The Dynamics of Capital Structure, Economics and Finance No. p. 333

3. Bhaduri S., (2002). N. Determinants of Capital Structure Choice: a Study of the Indian Sector. Applied Financial Economics, 2002, 12, p. 655-665

4. Bhamra S., Kuehn L., Strebulaev I., (2010). The Aggregate Dynamics of Capital Structure and Macroeconomic Risk, Review of Financial Studies 23, 12, p. 4187-4241

5. Céspedes J., Gonzaléz M., Molina C.A. Ownership and capital structure in Latin America. Journal of Business Research, 63, 2010, pp.248-254

6. Chakraborty I. Capital Structure in an emerging stock market: The case of India. Research in International Business and Finance, 24 (2010), 295-314

7. Chen J.J., (2004). Determinants of Capital Structure of Chinese-listed Companies. Economic Change and Restructuring, Vol. 38 (1), 2004, p. 1-35.

8. Dang, V.A, Kim, M., Shin, Y., (2012). Asymmetric Capital Structure Adjustments: New Evidence from Dynamic Panel Threshold Models. Journal of Empirical Finance, 19(4), p. 465-482.

9. Delcoure N., (2007). The determinants of capital structure in transition economies. International Review of Economics and Finance, 16 (2007), p. 400-415

10. Drobetz, Wolfgang, Wanzenried G., (2006). What determines the speed of adjustment to the target capital structure? Applied Financial Economics 16:13, p. 941-958.

11. Frank M., Goyal V., (2003). Testing the Pecking Order Theory of Capital Structure // Journal of Financial Economics. Vol. 67. N 2., p. 217-248.

12. Frank M.Z., Goyal V.K., (2008). Trade-Off and Pecking Order Theories of Debt. Handbook of Empirical Corporate Finance, Volume 2 Edited by B. Espen Eckbo, 2008, p. 135-202

13. Goldfeld S. M., Quandt R. E. A Markov model for switching regressions //Journal of econometrics. 
- 1973. - T. 1. - №. 1. - C. 3-15.

14. Hackbarth, D., Miao J., Morellec E., (2006). Capital structure, credit risk, and macroeconomic conditions, Journal of Financial Economics, 82, p. 519 -550.

15. Hess D., Immenkötter P., (2011). Optimal leverage, its benefits, and the business cycle. CFR Working Papers 11-12, University of Cologne

16. Mahakud J., Mukherjee S., (2011). Determinants of Adjustment Speed to Target Capital Structure: Evidence from Indian Manufacturing Firms, 2011 International Conference on Economics and Finance Research IPEDR vol.4

17. Mazur K., (2007). The determinants of capital structure choice: Evidence from Polish Companies, International Atlantic Economic Society, 13, p. 495-514

18. Mitton, T., (2008). Why Have Debt Ratios Increased for Firms in Emerging Markets? European Financial Management, Vol. 14, Issue 1, p. 127-151.

19. Nivorozhkin, E., (2002). Capital Structures in Emerging Stock Market: the Case of Hungary. The Developing Economies Volume 40, Number 2, p. 1-27

20. Öztekin Ö., Flannery M., (2012). Institutional determinants of capital structure adjustment speeds, Journal of Financial Economics 103 (1), p. 88-112

21. Pandey I., (2001). Capital Structure and the Firm Characteristics: Evidence from an Emerging Market, IIMA Working Paper No. 2001-10-04, p. 1-19.

22. Rajan G., Zingales L., (1995). What Do We Know About Capital Structure? Some Evidence from International Data, NBER Working Papers No. 4875, p. 1421-1460.

23. Mahfuzah Salim, Raj Yadav, Capital Structure and Firm Performance: Evidence from Malaysian Listed Companies, Procedia - Social and Behavioral Sciences, Volume 65, 3 December 2012, Pages 156-166, ISSN 1877-0428, http://dx.doi.org/10.1016/j.sbspro.2012.11.105.

24. Wiwattanakantang Y., (1999). An empirical study on the determinants of the capital structure of Thai firms. Pacific-Basin Finance Journal 7 (3-4), p. 371-403.

25. Kokoreva M, (2012). Capital Structure Choice in BRIC and Eastern Europe: Empirical Evidence. E-journal Corporate Finance. 2012. № 2., P. 58-70. 